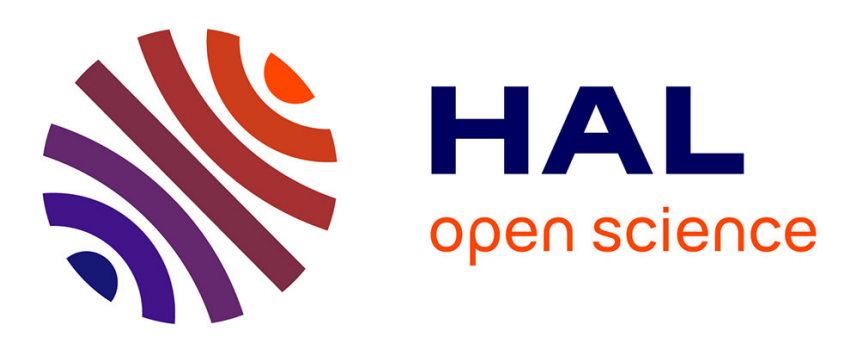

\title{
Stabilization of feedforward discrete-time dynamics through Immersion and Invariance
}

\author{
Salvatore Monaco, Dorothée Normand-Cyrot, Mattia Mattioni
}

\section{To cite this version:}

Salvatore Monaco, Dorothée Normand-Cyrot, Mattia Mattioni. Stabilization of feedforward discretetime dynamics through Immersion and Invariance. 2016 American Control Conference (ACC), Jul 2016, Boston, United States. pp.264 - 269, 10.1109/ACC.2016.7524926 . hal-01323560

\section{HAL Id: hal-01323560 \\ https://hal-centralesupelec.archives-ouvertes.fr/hal-01323560}

Submitted on 30 May 2016

HAL is a multi-disciplinary open access archive for the deposit and dissemination of scientific research documents, whether they are published or not. The documents may come from teaching and research institutions in France or abroad, or from public or private research centers.
L'archive ouverte pluridisciplinaire HAL, est destinée au dépôt et à la diffusion de documents scientifiques de niveau recherche, publiés ou non, émanant des établissements d'enseignement et de recherche français ou étrangers, des laboratoires publics ou privés. 


\title{
Stabilization of feedforward discrete-time dynamics through immersion and invariance
}

\author{
Salvatore Monaco, Dorothée Normand-Cyrot and Mattia Mattioni
}

\begin{abstract}
The paper deals with the problem of stabilizing discrete-time feedforward dynamics through Immersion and Invariance. Closed loop stabilization of the equilibrium is achieved making use of a passivity-based controller combined with a domination argument. A simulated example illustrates the performances.
\end{abstract}

Index Terms-Nonlinear output feedback; Stability of nonlinear systems; Lyapunov methods

\section{INTRODUCTION}

Forwarding is a stabilizing approach developed in continuous time (see [1], [2]) for dynamics admitting a particular cascaded (or triangular) structure. It provides a systematic bottom up recursive Lyapunov-based design procedure which can be interpreted as the dual of the celebrated back-stepping one [3]: instead of assuming a state component as a virtual control and controlling through forwarding, stabilization is achieved by iteratively adding a state component which "integrates" the other ones. Such an approach has been developed in discrete time in [4] to stabilize classes of nonlinear dynamics under Lyapunov-based bounded feedback.

In the present work we consider discrete-time strictfeedforward dynamics of the form

$$
\begin{aligned}
x_{j k+1} & =x_{j k}+F_{j}\left(x_{1 k}, x_{2 k}, \ldots, x_{j-1 k}, u_{k}\right) ; \quad j=2, \ldots, n \\
x_{1 k+1} & =F_{1}\left(x_{1 k}, u_{k}\right)
\end{aligned}
$$

where $x_{1} \in \mathbb{R}^{p}$ and the $x_{j} \in \mathbb{R}$ for $j=2, \ldots, n$ and $u \in U \subseteq$ $\mathbb{R}^{m}, m=1$. The proposed control strategy extends the design introduced in [5] for systems in strict-feedforward form (when setting $x_{1 k+1}=u_{k}, m=1$ ). Here, the general non linear $x_{1}$-dynamics is known to be stabilizable under a suitable state feedback but the knowledge of a control Lyapunov Function is not assumed. The problem is presently set in the context of Immersion and Invariance - I\&I. I\&I was proposed in [6]-[7] for stabilizing continuous-time systems and reformulated in [8]-[9] in discrete-time; the overall design results to be less demanding in such a context.

This work was partially supported by a CNRS-ST2I International Scientific Project - PICS - for cooperation between France and Italy.

S.Monaco is with Dipartimento di Ingegneria Informatica, Automatica e Gestionale, DIAG Università di Roma "La Sapienza", via Ariosto 25, 00185 Roma, Italy. salvatore.monaco@uniromal. it

D. Normand-Cyrot is with Laboratoire des Signaux et Systèmes (L2S, UMR CNRS 8506) CNRS - Centrale-Supelec; 3, rue Joliot Curie, 91192, Gif-sur-Yvette, France cyrotel2s. centralesupelec.fr

M. Mattioni is with both Laboratoire des Signaux et Systèmes (L2S, CNRS-CentraleSupelec-Univ. Paris-Sud) and Dipartimento di Ingegneria Informatica, Automatica e Gestionale, DIAG (Università di Roma "La Sapienza") mattia.mattioniel2s.centralesupelec.fr
With reference to (1), a preliminary controller ensuring global asymptotic stability - GAS - of the origin of the $x_{1}$ dynamics is computed so defining the target system. Then the design is completed for ensuring attractiveness of a certain invariant set associated with the target $x_{1}$-dynamics. Boundedness of the state trajectories guarantees global asymptotic stabilization of the closed-loop equilibrium. Attractiveness of the invariant set is achieved thanks to passivity arguments and negative output feedback as proposed in [10]. As usual in a discrete-time context, the negative output feedback is only implicitly defined; for this reason, domination arguments as developed in [11]-[4] are used to provide an explicit bounded solution.

The paper is organized as follows. Some preliminaries on discrete-time state space representations and I\&I stabilizability are in Section II. A brief motivating discussion is in Section III. The proposed control design is developed for the elementary feedforward two block dynamics in Section IV. An example concludes the paper in Section V.

\section{RECALLS AND PRELIMINARIES}

\section{A. The Differential Difference Representation}

Following [12], nonlinear discrete-time dynamics in the form of a map $x_{k+1}=\mathscr{F}\left(x_{k}, u_{k}\right)$ with $\mathscr{F}(\cdot, u)$ a $\mathbb{R}^{n}$-valued smooth map, smootly parameterized by $u \in U$, can be represented as a couple of a difference and a differential equation

$$
\begin{aligned}
x^{+} & =\mathscr{F}_{0}(x) \\
\frac{\partial x^{+}(u)}{\partial u} & =\mathscr{G}\left(x^{+}(u), u\right)
\end{aligned}
$$

where $x^{+}(u)$ represents a curve in $\mathbb{R}^{n}$ parameterized by $u$, $\mathscr{F}_{0}(x):=\mathscr{F}(x, 0):=x^{+}(0)$, defines the initial condition of the differential equation $(3) . \mathscr{G}(\cdot, u)$ on $\mathbb{R}^{n}$, parameterized by $u$, is computed to satisfy the equality

$$
\mathscr{G}(\mathscr{F}(x, u), u)=\frac{\partial \mathscr{F}(x, u)}{\partial u} .
$$

Given $\mathscr{F}(\cdot, u)$, the existence of $\mathscr{G}(\cdot, u)$ is ensured by the reversibility of $\mathscr{F}_{0}(\cdot)$ as a function of $x$, so uniquely defining and computing $\mathscr{G}(\cdot, u)$ for $u$ sufficiently small as

$$
\mathscr{G}(x, u):=\left.\frac{\partial \mathscr{F}(\cdot, u)}{\partial u}\right|_{x=\mathscr{F}^{-1}(x, u)}
$$

where $\mathscr{F}^{-1}(x, u)$ denotes the reverse function, i.e., satisfying $\mathscr{F}\left(\mathscr{F}^{-1}(x, u), u\right)=x$.

For any $x$, completeness of the vector field $\mathscr{G}(\cdot, u)$ for all $u \in U$ ensures integrability of (3) so recovering the usual 
representation in the form of a map

$$
x^{+}(u)=\mathscr{F}(x, u)=x^{+}(0)+\int_{0}^{u} \mathscr{G}\left(x^{+}(v), v\right) \mathrm{d} v
$$

with $x^{+}(0):=\mathscr{F}_{0}(x)$. Consequently, given any smooth function $\mathscr{H}: \mathbb{R}^{n} \rightarrow \mathbb{R}$, its variation with respect to $u$ around $\mathscr{H}\left(x^{+}(0)\right)$ admits the integral form representation

$$
\mathscr{H}(\mathscr{F}(x, u))-\mathscr{H}\left(\mathscr{F}_{0}(x)\right)=\int_{0}^{u} \mathrm{~L}_{\mathscr{G}(\cdot, v)} \mathscr{H}\left(x^{+}(v)\right) \mathrm{d} v
$$

where $\mathrm{L}_{\mathscr{G}(\cdot, \cdot)} \mathscr{H}(\cdot)$ represents the Lie derivative of $\mathscr{H}(\cdot)$ along the vector field $\mathscr{G}(\cdot, \cdot)$; i.e.

$$
\mathrm{L}_{\mathscr{G}(\cdot, v)} \mathscr{H}\left(x^{+}(v)\right)=\left[\frac{\partial \mathscr{H}(\cdot)}{\partial x} \mathscr{G}(\cdot, v)\right]_{x^{+}(v)} .
$$

(2) and (3) define the $\left(\mathscr{F}_{0}, \mathscr{G}\right)$ representation of a discretetime dynamics.

\section{B. $\left(\mathscr{F}_{0}, \mathscr{G}\right)$ representation of feedforward dynamics}

According to these definitions it is a matter of computations to verify that any nonlinear feedforward dynamics of the form (1), with $F_{1}(x, u)$ reversible in $x_{1}$ for all $u \in U$, admits the following $\left(\mathscr{F}_{0}, \mathscr{G}\right)$ representation

$$
\begin{aligned}
x_{j}^{+} & =x_{j}+F_{j 0}\left(x_{j-1}, \ldots, x_{1}\right) ; \quad j=2, \ldots, n \\
x_{1}^{+} & =F_{10}\left(x_{1}\right) \\
\frac{\partial x_{j}^{+}(u)}{\partial u} & =G_{j}\left(x_{j-1}^{+}(u), \ldots, x_{1}^{+}(u), u\right) ; j=2, \ldots, n \\
\frac{\partial x_{1}^{+}(u)}{\partial u} & =G_{1}\left(x_{1}^{+}(u), u\right)
\end{aligned}
$$

with

$$
\begin{aligned}
& \mathscr{F}_{0}(x)=\operatorname{col}\left[x_{n}+F_{n 0}\left(x_{n-1}, \ldots, x_{1}\right), \ldots, x_{2}+F_{20}\left(x_{1}\right), F_{10}\left(x_{1}\right]\right. \\
& \mathscr{F}_{0}(0)=0 \text { and } \mathscr{G}(\cdot, u):=\operatorname{col}\left[G_{n}(\cdot, u), \ldots, G_{1}(\cdot, u)\right] .
\end{aligned}
$$

For, it is sufficient to verify that reversibility of $F_{1}\left(x_{1}, u\right)$ in $x_{1}$ is sufficient to imply reversibility of $\mathscr{F}_{0}(x)$ in $x$. More precisely, the reverse dynamics can be iteratively computed so getting

$$
\begin{aligned}
x_{1} & =F_{1}^{-1}\left(x_{1}^{+}(u), u\right) \\
x_{2}= & x_{2}^{+}(u)-F_{2}\left(x_{1}, u\right)=x_{2}^{+}(u)-F_{2}\left(F_{1}^{-1}\left(x_{1}^{+}(u), u\right), u\right) \\
x_{3}= & x_{3}^{+}(u)-F_{3}\left(x_{2}^{+}(u)-\right. \\
& \left.\quad F_{2}\left(F_{1}^{-1}\left(x_{1}^{+}(u), u\right), u\right), F_{1}^{-1}\left(x_{1}^{+}(u), u\right), u\right) \\
& \ldots
\end{aligned}
$$

Then, according to (4), one computes for $j=2, \ldots, n$, the control vector fields $G_{j}\left(x_{j-1}^{+}(u), \ldots, x_{1}^{+}(u), u\right)$ as

$$
\begin{aligned}
G_{j}\left(x_{j-1}, \ldots, x_{1}, u\right) & =\left.\frac{\partial F_{j}\left(x_{j-1}, \ldots, x_{1}, u\right)}{\partial u}\right|_{x=\mathscr{F}-1}(x, u) \\
G_{1}\left(x_{1}, u\right) & =\left.\frac{\partial F_{1}\left(x_{1}, u\right)}{\partial u}\right|_{x_{1}=F_{1}^{-1}\left(x_{1}, u\right)}
\end{aligned}
$$

which maintain the required triangular form.

In the sequel, the design is instrumentally developed with reference to strict-feedforward dynamics which admit the $\left(\mathscr{F}_{0}, \mathscr{G}\right)$ representation $(5,6)$, but the solution can be applied to a discrete-time system of the form (1).

\section{The discrete-time I\&I stabilizability conditions}

Following [6], let us preliminarily formulate I\&I stabilization for generally nonlinear difference equations [9].

Theorem 2.1: Consider the nonlinear discrete-time dynamics

$$
x_{k+1}=\mathscr{F}\left(x_{k}, u_{k}\right)
$$

with state $x \in \mathbb{R}^{n}$, control $u \in \mathbb{R}$ and let $x^{*} \in \mathbb{R}^{n}$ the equilibrium to be stabilized. Let $p<n$ and assume that we can find mappings

$$
\begin{array}{lll}
\alpha(\cdot) & : \quad \mathbb{R}^{p} \rightarrow \mathbb{R}^{p} ; \quad \pi(\cdot): \mathbb{R}^{p} \rightarrow \mathbb{R}^{n} \\
\phi(\cdot) & : \quad \mathbb{R}^{n} \rightarrow \mathbb{R}^{n-p} ; \quad \psi(\cdot, \cdot): \mathbb{R}^{n \times(n-p)} \rightarrow \mathbb{R}
\end{array}
$$

such that the following hold.

$\mathbf{H}_{1 d} \quad$ (Target System) - The dynamics with state $\xi \in \mathbb{R}^{p}$

$$
\xi_{k+1}=\alpha\left(\xi_{k}\right)
$$

has a globally asymptotically stable equilibrium at $\xi^{*} \in \mathbb{R}^{p}$ and $x^{*}=\pi\left(\xi^{*}\right)$.

$\mathbf{H}_{2 d} \quad$ (Immersion and invariance condition) - For all $\xi \in$ $\mathbb{R}^{p}$, there exists $c(\cdot): \mathbb{R}^{p} \rightarrow \mathbb{R}$ such that

$$
\mathscr{F}(\pi(\xi), c(\xi))=\pi(\alpha(\xi)) .
$$

$\mathbf{H}_{3 d}$ (Implicit manifold) - The following identity between sets holds

$\left\{x \in \mathbb{R}^{n} \mid \phi(x)=0\right\}=\left\{x \in \mathbb{R}^{n} \mid x=\pi(\xi)\right.$ for $\left.\xi \in \mathbb{R}^{p}\right\}$

$\mathbf{H}_{4 d} \quad$ (Manifold attractivity and trajectory boundedness) - All the trajectories of the system

$$
\begin{aligned}
& x_{k+1}=\mathscr{F}\left(x_{k}, \psi\left(x_{k}, z_{k}\right)\right) \\
& z_{k+1}=\phi\left(\mathscr{F}\left(x_{k}, \psi\left(x_{k}, z_{k}\right)\right)\right)
\end{aligned}
$$

with $z \in \mathbb{R}^{n-p}, z_{0}=\phi\left(x_{0}\right)$, are bounded for all $k \geq 0$ and satisfy $\lim _{k \rightarrow \infty} z_{k}=0$ and $\left.\psi(\cdot, 0)\right|_{x=\pi(\xi)}=c(\xi)$.

Then, $x^{*}$ is a globally asymptotically stable equilibrium of the closed loop dynamics $x_{k+1}=\mathscr{F}\left(x_{k}, \psi\left(x_{k}, \phi\left(x_{k}\right)\right)\right)$.

Definition 2.1: The discrete-time nonlinear dynamics (7) is said to be I\&I-stabilizable with target dynamics $\xi_{k+1}=$ $\alpha\left(\xi_{k}\right)$ when $\mathbf{H}_{1 d}-\mathbf{H}_{2 d}-\mathbf{H}_{3 d}-\mathbf{H}_{4 d}$ in Theorem 2.1 are satisfied.

We show in this paper that I\&I stabilization of feedforward dynamics reduces the control design to the iterative stabilization of suitably defined one-dimensional dynamics.

\section{A MOTIVATING DiscUSSION}

To briefly discuss the idea behind forwarding, let the elementary discrete-time cascade-connected dynamics

$$
y_{k+1}=y_{k}+H\left(x_{k}\right) ; \quad x_{k+1}=F\left(x_{k}\right)
$$

where $x \in \mathbb{R}^{p}, y$ is scalar, $H$ and $F$ are continuous functions and the origin of the $x$-subsystem is assumed asymptotically stable; i.e. there exists a positive definite Lyapunov function $V(x)$ such that $V\left(x_{k+1}\right)-V\left(x_{k}\right)<0$ for all $x_{k} \neq 0$. The existence of an invariant stable set described by the graph of a function $y=\Phi(x)$, implies the stability of the cascade. 
In fact, if such a function $\Phi(x)$ (with $\Phi(0)=0$ ) exists, it satisfies the invariance implication

$$
\left(y_{0}, x_{0}\right) \in \Omega=\{(y, x) \text { s.t. } y=\Phi(x)\} \Rightarrow\left(y_{k}, x_{k}\right) \in \Omega ; \forall k>0
$$

for any $\left(y_{k}, x_{k}\right)$ solutions to the cascade system. $\Phi(\cdot)$ can be computed by solving the equality

$$
\Phi(F(x))-\Phi(x)=H(x)
$$

with initial condition $\Phi(0)=0$. On these bases, it is easily verified that a Lyapunov function for the overall system is given by $W(x, y)=V(x)+\frac{1}{2}(y-\Phi(x))^{2}$ with first order increment $\Delta_{k} W=W\left(x_{k+1}, y_{k+1}\right)-W\left(x_{k}, y_{k}\right)$ negative definite equal to $\Delta_{k} V=V\left(F\left(x_{k}\right)\right)-V\left(x_{k}\right)$.

Forwarding relies on this basic idea to stabilize controlled cascade-connected dynamics of the form

$$
y_{k+1}=y_{k}+H\left(x_{k}\right), \quad x_{k+1}=F\left(x_{k}, u_{k}\right) .
$$

In this case, according to the $\left(F_{0}, G\right)$ representation of the $x$-dynamics

$$
x_{k}^{+}\left(u_{k}\right):=F\left(x_{k}, u_{k}\right)=F_{0}\left(x_{k}\right)+\int_{0}^{u_{k}} \mathrm{~L}_{G(\cdot, v)}\left(x^{+}(v)\right) \mathrm{d} v
$$

with $F_{0}(x):=F(x, 0)$, one has

$$
\begin{aligned}
\Delta_{k} W & =V\left(F\left(x_{k}\right)\right)-V\left(x_{k}\right)+\int_{0}^{u_{k}} \mathrm{~L}_{G(\cdot, v)} V\left(x^{+}(v)\right) \mathrm{d} v \\
& +\frac{1}{2}\left(\int_{0}^{u_{k}} \mathrm{~L}_{G(\cdot, v)} \Phi\left(x^{+}(v)\right) \mathrm{d} v\right)^{2} \\
& -\left(y_{k}-\Phi\left(x_{k}\right)\right) \int_{0}^{u_{k}} \mathrm{~L}_{G(\cdot, v)} \Phi\left(x^{+}(v)\right) \mathrm{d} v
\end{aligned}
$$

It clearly comes out that choosing $u_{k}$ to render negative $\Delta_{k} W$ in (13) is a difficult task since it involves solving an implicit inequality in $u_{k}$.

An equivalent solution, which recalls the structure of the continuous-time one, can be obtained by rewriting $\Delta_{k} W$ as

$$
\begin{aligned}
\Delta_{k} W & =V\left(F\left(x_{k}\right)\right)-V\left(x_{k}\right)+u_{k} \int_{0}^{1} \mathrm{~L}_{G\left(\cdot, s u_{k}\right)} V\left(x^{+}\left(s u_{k}\right)\right) \mathrm{d} s \\
& +\frac{u_{k}^{2}}{2}\left(\int_{0}^{1} \mathrm{~L}_{G\left(\cdot, s u_{k}\right)} \Phi\left(x^{+}\left(s u_{k}\right)\right) \mathrm{d} s\right)^{2} \\
& -u_{k}\left(y_{k}-\Phi\left(x_{k}\right)\right) \int_{0}^{1} \mathrm{~L}_{G\left(\cdot, s u_{k}\right)} \Phi\left(x^{+}\left(s u_{k}\right)\right) \mathrm{d} s
\end{aligned}
$$

and solving the implicit equality below

$u=-\Gamma^{-1} \int_{0}^{1}\left[\left.\mathrm{~L}_{G(\cdot, s u)} V\right|_{x^{+}(s u)}-\left.(y-\Phi(x)) \mathrm{L}_{G(\cdot, s u)} \Phi\right|_{x^{+}(s u)}\right] \mathrm{d} s$

with positive gain function

$$
\Gamma=\left[1+\frac{\left[\left.\int_{0}^{1} \mathrm{~L}_{G(\cdot, s u)} \Phi\right|_{x^{+}(s u)} \mathrm{d} s\right]^{2}}{2}\right] .
$$

Remark 3.1: The control solution (14) recalls the continuous-time stabilizing control

$$
u=-\left[\mathrm{L}_{g} V(x)-(y-\phi(x)) \mathrm{L}_{g} \phi(x)\right]
$$

which ensures global asymptotic stabilization of the system

$$
\dot{y}=h(x), \quad \dot{x}=f(x)+u g(x)
$$

when the origin of the $x$-subsystem is assumed asymptotically stable (there exists a positive definite Lyapunov function $V(x)$ such that $\mathrm{L}_{f} V<0$ for all $x \neq 0$ ) and when the function $\phi(x)$ is computed to satisfy $\mathrm{L}_{f} \phi=h$ with $\phi(0)=0$.

It will be shown in the sequel that the concept of I\&I stabilization combined with a domination argument makes the design constructive in discrete-time too.

\section{I\&I FORWARDING STABILIZATION}

Consider the following elementary feedforward dynamics over $\mathbb{R}^{p+1}$

$$
x_{2 k+1}=x_{2 k}+F_{2}\left(x_{1 k}, u_{k}\right), \quad x_{1 k+1}=F_{1}\left(x_{1 k}, u_{k}\right)
$$

and assume that:

A1) - the origin of the $x_{1}$-subsystem is asymptotically stable, i.e. there exists a positive definite Lyapunov function $V(\cdot)$ : $\mathbb{R}^{p} \rightarrow \mathbb{R}$ such that $V\left(F_{1}\left(x_{1 k}, 0\right)\right)-V\left(x_{1 k}\right)<0$ for all $x_{1 k} \neq 0$; A2) - there exists a function $\Phi_{1}\left(x_{1}\right)$ with $\Phi_{1}(0)=0$ satisfying the following equality

$$
\Phi_{1}\left(F_{10}\left(x_{1}\right)\right)-\Phi_{1}\left(x_{1}\right)=F_{20}\left(x_{1}\right)
$$

with $F_{10}\left(x_{1}\right)=F_{1}\left(x_{1}, 0\right)$ and $F_{20}\left(x_{1}\right)=F_{20}\left(x_{1}, 0\right)$.

The $\left(F_{0}, G\right)$ representation of (18) takes the form:

$$
\begin{aligned}
& x_{2}^{+}=x_{2}+F_{20}\left(x_{1}\right), \quad \frac{\partial x_{2}^{+}(u)}{\partial u}=G_{2}\left(x_{1}^{+}(u), u\right) \\
& x_{1}^{+}=F_{10}\left(x_{1}\right), \quad \frac{\partial x_{1}^{+}(u)}{\partial u}=G_{1}\left(x_{1}^{+}(u), u\right)
\end{aligned}
$$

with

$$
G_{1}\left(F_{1}\left(x_{1}, u\right), u\right)=F_{1 u}^{\prime}(x, u) ; G_{2}\left(F_{1}\left(x_{1}, u\right), u\right)=F_{2 u}^{\prime}\left(x_{1}, u\right) .
$$

Setting $z_{2}=x_{2}-\Phi_{1}\left(x_{1}\right)$ and

$$
G_{z}(\cdot, u):=G_{2}(\cdot, u)-\mathrm{L}_{G_{1}(\cdot, u)} \Phi_{1}(\cdot)
$$

with $\mathrm{L}_{G_{1}(\cdot, u)} \Phi_{1}\left(F_{1}\left(x_{1}, u\right)\right):=\frac{\partial \Phi_{1}}{\partial x_{1}}\left(F_{1}\left(x_{1}, u\right)\right) F_{1 u}^{\prime}\left(x_{1}, u\right)$, the following result holds true.

Proposition 4.1: - Given the discrete-time feedforward dynamics (18) satisfying A1) A2), then the equilibrium is I\&I stabilizable with target dynamics $\xi_{k+1}=F_{10}\left(\xi_{1 k}\right)$.

Proof: For, it is sufficient to show that the $\mathbf{H}_{1 d}$ to $\mathbf{H}_{3 d}$ conditions in Theorem 2.1 are satisfied. First, setting $x_{1}=\xi$, the target dynamics is defined by the $x_{1}$-dynamics in free evolution because of assumption A1) (i.e. $\xi_{k+1}=F_{10}\left(\xi_{1 k}\right)$ ). Then, the immersion mapping $\pi(\xi)$ is defined from $\mathbb{R}^{p}$ to $\mathbb{R}^{p+1}$ as $\pi(\xi)=\operatorname{col}\left(\Phi_{1}(\xi), \xi\right)$ so immediately verifying the invariance condition (9) because of assumption A2) with $c(\xi)=0$. Setting now $z_{2}=x_{2}-\Phi_{1}\left(x_{1}\right)$, one expresses with (21) the $z_{2}$-dynamics, driftless by construction of $\Phi_{1}$; i.e.

$$
\begin{aligned}
z_{2}^{+} & =z_{2} \\
\frac{\partial z_{2}^{+}(u)}{\partial u} & =G_{z}\left(x_{1}^{+}(u), u\right) .
\end{aligned}
$$

By construction, the $p$-dimensional set described by $z_{2}=0$ is invariant and the I\&I design reduces to find a control that makes such set attractive while guaranteeing boundedness of the closed loop trajectories.

The control design is discussed below. 


\section{A. I\&I dead-beat stabilizing control}

A first dead-beat solution can be computed by solving in $u_{k}$ for all $z_{2 k}$, the implicit equality $z_{2 k+1}=0$; i.e.

$$
z_{2 k}+u_{k} \int_{0}^{1}\left(G_{2}\left(\cdot, s u_{k}\right)-\mathrm{L}_{G_{1}\left(\cdot, s u_{k}\right)} \Phi_{1}\right)\left(x_{1}^{+}\left(s u_{k}\right)\right) \mathrm{d} s=0
$$

so bringing in one step $z_{2 k+1}$ to zero (equivalently $x_{2 k+1}$ to $\left.\Phi\left(x_{1 k+1}\right)\right)$. Then $u_{k+i}=0$ for $i \geq 1$ guarantees $z_{2 k+i+1}=0$ so that the trajectory lays on the stable set.

\section{B. I\&I negative output feedback}

It is shown in this section that the concept of u-average passivity, introduced by the authors in [10], can be fruitfully used to get asymptotic stabilization of the origin of the $z_{2}$ dynamics. With this in mind, let us associate to (20) in the coordinates $\left(x_{1}, z_{2}\right)$ the output

$$
H\left(x_{1}, z_{2}, u\right)=\mathrm{L}_{G_{z}\left(x_{1}, u\right)} V_{z}\left(z_{2}\right)
$$

ant its average [10]

$$
H_{a v}^{+}\left(x_{1}, z_{2}, u\right)=\frac{1}{u} \int_{0}^{u} H\left(x_{1}^{+}(v), z_{2}^{+}(v), v\right) \mathrm{d} v .
$$

The next result is an immediate consequence of the driftless property of (22).

Proposition 4.2: The feedforward dynamics (18) satisfying A1)-A2) with output (23) is u-average lossless with storage function $V_{z}\left(z_{2}\right)=\frac{1}{2} z_{2}^{2}$; i.e. it satisfies

$$
V_{z}\left(z_{2 k+1}\right)-V_{z}\left(z_{2 k}\right)=\int_{0}^{u_{k}} H\left(x_{1}^{+}(v), z_{2}^{+}(v), v\right) \mathrm{d} v .
$$

In the present case, one easily computes with $G_{z}(\cdot, u)$ in (21)

$$
\begin{aligned}
H\left(x_{1}^{+}(u), z_{2}^{+}(u), u\right) & =z_{2}^{+}(u) G_{z}\left(x_{1}^{+}(u), u\right) \\
H_{a v}^{+}\left(x_{1}, z_{2}, u\right) & =\int_{0}^{1} z_{2}^{+}(s u) G_{z}\left(x_{1}^{+}(s u), s u\right) \mathrm{d} s .
\end{aligned}
$$

From [10], any feedback law making (23) negative renders the origin of the $z_{2}$-dynamics GAS, provided the so defined output $H\left(x_{1}, z_{2}, u\right)$ is zero state detectable - ZSD -; i.e.

ZSD: no solution of the uncontrolled dynamics (18) can stay in the set $\left\{\left(x_{1}, z_{2}\right) \in \mathbb{R}^{p+1}\right.$ s.t $H_{a v}^{+}\left(x_{1}, z_{2}, 0\right)=$ $\left.z_{2} G_{z}\left(F_{10}\left(x_{1}\right), 0\right)\right\}$ other than solutions converging asymptotically to the zero equilibrium.

Proposition 4.3 (I\&I Negative u-average output feedback): Given the feedforward dynamics (18) satisfying A1) A2) with output mapping $z_{2}^{+}(u) G_{z}\left(x_{1}^{+}(u), u\right)$ assumed zero state detectable then, for all $\left(x_{1}, z_{2}\right) \in \mathbb{R}^{p+1}$, the control law $u$ solution of the algebraic equation

$u=-\varepsilon\left(x_{1}\right) z_{2}\left[1+\frac{1}{2}\left(\int_{0}^{1} G_{z}\left(x_{1}^{+}(s u), s u\right) \mathrm{d} s\right)^{2}\right]^{-1} \int_{0}^{1} G_{z}\left(x_{1}^{+}(s u), s u\right) \mathrm{d} s$

with sufficiently small $\varepsilon\left(x_{1}\right)>0$, ensures global asymptotic stabilization of the origin of (18).

Proof: From the lossless equality (23) rewritten as

$$
V_{z}\left(z_{2 k+1}\right)-V_{z}\left(z_{2 k}\right)=u_{k} H_{a v}^{+}\left(x_{1 k}, z_{2 k}, u_{k}\right)
$$

and according to Proposition 4.2, the control law solution of the algebraic equality

$$
\begin{aligned}
& u=-\varepsilon\left(x_{1}\right) H_{a v}^{+}\left(x_{1}, z_{2}, u\right)= \\
& -\varepsilon\left(x_{1}\right)\left[z_{2} \int_{0}^{1} G_{z}\left(x_{1}^{+}(s u), s u\right) \mathrm{d} s-\frac{u}{2}\left[\int_{0}^{1} G_{z}\left(x_{1}^{+}(s u), s u\right) \mathrm{d} s\right]^{2}\right]
\end{aligned}
$$

with sufficiently small $\varepsilon\left(x_{1}\right)>0$ achieves in closed loop

$$
V_{z}\left(z_{2 k+1}\right)-V_{z}\left(z_{2 k}\right)=-\varepsilon\left(x_{1}\right)\left[H_{a v}^{+}\left(x_{1 k}, z_{2 k}, u_{k}\right)\right]^{2} \leq 0 .
$$

Asymptotic stability to the origin of the $z_{2}$-dynamics follows under the requested zero state detectability condition of the mapping $z_{2} G_{z}\left(F_{10}\left(x_{1}\right), 0\right)$. Boundedness of the trajectories is ensured by sufficiently small $\varepsilon\left(x_{1}\right)$. Then, I\&I stabilization of the origin of the feedforward dynamics (18) follows.

\section{A constructive bounded solution}

The solution proposed in (24) is implicitly defined and thus cannot be exactly computed in general. Setting $s=0$ in the right hand side of (24), one gets a computable approximation of the solution in the form

$$
u=-\varepsilon\left(x_{1}\right) z_{2}\left[1+\frac{1}{2}\left[G_{z}\left(F_{10}\left(x_{1}\right), 0\right)\right]^{2}\right]^{-1} G_{z}\left(F_{10}\left(x_{1}\right), 0\right)
$$

with $G_{z}\left(F_{10}\left(x_{1}\right), 0\right)=G_{2}\left(F_{10}\left(x_{1}\right), 0\right)-\mathrm{L}_{G^{1}(\cdot, 0)} \Phi_{1}\left(F_{10}\left(x_{1}\right)\right)$. Lemma 4.1 below, recalled from [11], [4] is used to provide a bounded feedback of the form (26) with suitably chosen gain.

Lemma 4.1: [11] Let $K(\xi, u)$ be a continuous function. For any strictly positive real number $\bar{u}$, there exists a function $\lambda(\xi)$, as smooth as $K(\xi, u)$ is, such that if $u(\xi)=$ $-\lambda(\xi) K(\xi, u)$ then, for all $\xi$, we have

$$
|u(\xi)| \leq \bar{u} \text { and } u(\xi) K(\xi, u) \leq \frac{1}{2} \lambda(\xi)|K(\xi, 0)|^{2} .
$$

Moreover, if $K(\xi, u)$ is $C^{1}$, then $\lambda$ is strictly positive on any compact set.

On the basis of Proposition 4.3 and Lemma 4.1 we can now prove the following result

Theorem 4.1: Bounded I\&I negative u-average output feedback - Given the feedforward dynamics (18) satisfying A1)-A2) then, for any bound $\mu>0$, the feedback

$$
u\left(x_{1}, z_{2}\right)=-\varepsilon\left(x_{1}\right) \lambda\left(x_{1}, z_{2}\right) z_{2} G_{z}\left(F_{10}\left(x_{1}\right)\right)
$$

where $\lambda\left(x_{1}, z_{2}\right)$ is any function that satisfies

$0<\varepsilon\left(x_{1}\right) \lambda\left(x_{1}, z_{2}\right) \leq \frac{\mu}{(2 \mu+1)\left(1+\left|z_{2} G_{z}\left(F_{10}\left(x_{1}\right)\right)\right|\right)} \min \{1, C\}$

with

$$
C:=\min _{|u| \leq \frac{1}{2}}\left\{\frac{2}{\left[\int_{0}^{1} G_{z}\left(x_{1}^{+}(s u), s u\right) \mathrm{d} s\right]^{2}}\right\}
$$

and $\varepsilon\left(x_{1}\right)>0$ sufficiently small, ensures global asymptotic stabilization of the origin of (18) provided the set $\left\{x_{1} \in\right.$ $\mathbb{R}^{p}$ s.t. $\left.G_{z}\left(F_{10}\left(x_{1}\right)\right)=0\right\}$ is made of isolated points.

Proof: From Lemma 4.1, the feedback law (27) with

$$
C:=\min _{|u| \leq \frac{1}{2}}\left\{\frac{|u|}{\left|\int_{0}^{1} z_{2}^{+}(s u) G_{z}\left(x_{1}^{+}(s u), s u\right) \mathrm{d} s-z_{2} G_{z}\left(F_{10}\left(x_{1}\right)\right)\right|}\right\}
$$


rewritten as in (29) is bounded and guarantees negativity of $u H_{a v}^{+}\left(x_{1}, z_{2}, u\right)$; i.e. for all $\left(x_{1}, z_{2}\right)$, one has $\left|u\left(x_{1}, z_{2}\right)\right| \leq \mu$ and

$$
\int_{0}^{u} z_{2}^{+}(v) G_{z}\left(x_{1}^{+}(v), v\right) \mathrm{d} v \leq-\frac{1}{2} \varepsilon\left(x_{1}\right) \lambda\left(x_{1}, z_{2}\right)\left|z_{2} G_{z}\left(F_{10}\left(x_{1}\right)\right)\right|^{2} .
$$

When $z_{2}=0, u\left(x_{1}, 0\right)=0$. Boundedness of the whole state trajectories follows from Proposition 4.3 with sufficiently small $\varepsilon\left(x_{1}\right)>0$.

Remark 4.1: Theorem 4.1 can be used repeatedly to deduce that the feedforward system (1) is globally asymptotically stabilizable under A1) and successive conditions of the type A2). At each step of the design, the closed loop $\operatorname{col}\left(x_{1}, z_{2}\right)$-dynamics defines a new stable $\bar{x}_{1}$-dynamics in $\mathbb{R}^{p+1}$ which defines a new target dynamics and one adds an integrating variable $z_{3}=x_{3}-\bar{\Phi}\left(\bar{x}_{1}\right)$ which satisfies the invariance condition $\bar{\Phi}\left(\bar{F}_{10}\left(\bar{x}_{1}\right)\right)=\bar{F}_{30}\left(\bar{x}_{1}\right)$. This is repeated for the $n$ blocks.

Remark 4.2: The choice of the gain $\lambda\left(x_{1}, z_{2}\right)$ in (27) can be made (within the suitable interval) according to several strategies. In particular, it can be chosen so as to obtain robustness of the closed-loop system under the nominal control with respect to parameters uncertainty and external disturbances. In this case, suitable Lipschitz-like assumptions should be introduced for the mappings $F_{i}(i=1,2)$.

Remark 4.3: The same approach can be pursued when relaxing assumption A1) to the existence of a preliminary stabilizing feedback $c\left(x_{1}\right)$ for the $p$-dimensional $x_{1}$-dynamics. Then, the same result holds when substituting the dynamics $F_{10}\left(x_{1}\right)$ with $F_{1}\left(x_{1}, c\left(x_{1}\right)\right)$ and $F_{20}\left(x_{1}\right)$ with $F_{2}\left(x_{1}, c\left(x_{1}\right)\right)$.

Remark 4.4: Consider the continuous-time cascade

$$
\dot{x}_{2}=f_{2}\left(x_{1}\right)+u g_{2}\left(x_{1}\right) ; \quad \dot{x}_{1}=f_{1}\left(x_{1}\right)+u g_{1}\left(x_{1}\right)
$$

with the origin of the $x_{1}$-subsystem asymptotically stable (there exists a positive definite Lyapunov function $V\left(x_{1}\right)$ such that $\mathrm{L}_{f_{1}} V\left(x_{1}\right)<0$ for all $\left.x_{1} \neq 0\right)$ and assume the existence of the function $\phi_{1}\left(x_{1}\right)$ solving $\mathrm{L}_{f_{1}} \phi_{1}=f_{2}$ with $\phi_{1}(0)=0$. Under piecewise constant control over time intervals of length $\delta$, the sampled-data equivalent model takes the form (2)

$$
x_{2 k+1}=x_{2 k}+F_{2}^{\delta}\left(x_{1 k}, u_{k}\right) ; \quad x_{1 k+1}=F_{1}^{\delta}\left(x_{1 k}, u_{k}\right)
$$

with

$$
\begin{aligned}
F_{2}^{\delta}\left(x_{1 k}, u_{k}\right) & =\left.\int_{0}^{\delta} e^{\tau\left(f_{1}+u_{k} g_{1}\right)}\left(f_{2}+u_{k} g_{2}\right)\left(x_{1}\right) \mathrm{d} \tau\right|_{x_{1 k}} \\
F_{1}^{\delta}\left(x_{1 k}, u_{k}\right) & =\left.e^{\delta\left(f_{1}+u_{k} g_{1}\right)} x_{1}\right|_{x_{1 k}} .
\end{aligned}
$$

It is a matter of computations to show that the two conditions A1)-A2) hold with $\Phi_{1}(\cdot)=\phi_{1}(\cdot)$; which proves the existence of a piecewise constant solution from sampled state measures.

\section{EXAMPLE}

Let the academic example on the plane

$$
\dot{x}_{2}(t)=x_{1}(t)+x_{1}^{3}(t) ; \quad \dot{x}_{1}(t)=-x_{1}(t)+u(t) .
$$

It is easily verified that continuous-time I\&I stabilization is achieved by the control $u=-K z_{2}\left(1+x_{1}^{2}\right)$ with $K>0$, $z_{2}=x_{2}-\phi_{1}\left(x_{1}\right)$ and $\phi_{1}\left(x_{1}\right)=-x_{1}-\frac{1}{3} x_{1}^{3}$. Furthermore, the dynamics is zero state detectable with respect to the "output" map $z_{2}\left(1+x_{1}^{2}\right)$. Consider now the sampled-data equivalent to (31) which is exactly computable and given by

$$
\begin{aligned}
x_{2 k+1} & =x_{2 k}+\left(1-e^{-\delta}\right) x_{1 k}+x_{1 k}^{3} \int_{0}^{\delta} e^{-3 \tau} d \tau \\
& +u_{k}\left[e^{-\delta}+\delta-1+3 x_{1 k}^{2} \int_{0}^{\delta} e^{-2 \tau}\left(1-e^{-\tau}\right) d \tau\right] \\
& +3 x_{1 k} u_{k}^{2} \int_{0}^{\delta} e^{-\tau}\left(1-e^{-\tau}\right)^{2} d \tau+u_{k}^{3} \int_{0}^{\delta}\left(1-e^{-\tau}\right)^{3} d \tau \\
x_{1 k+1} & =e^{-\delta} x_{1 k}+\left(1-e^{-\delta}\right) u_{k} .
\end{aligned}
$$

The origin is still GAS for the discrete-time $x_{1}$-dynamics. Hence, one defines the discrete-time target as $\xi_{k+1}=e^{-\delta} \xi_{k}$. Then, setting $z_{2}=x_{2}-\phi_{1}\left(x_{1}\right)$ with the same $\phi_{1}\left(x_{1}\right)$ as in the continuous-time case, one verifies that $z_{2 k+1}=z_{2 k}$ under $u_{k} \equiv 0$. Consequently, the immersion mapping is described, as in the continuous-time case, by $\pi(\xi)=\operatorname{col}\left(-\xi-\frac{1}{3} \xi^{3}, \xi\right)$. The $z_{2}$-dynamics rewrites in the $\left(F_{0}, G\right)$ form as

$$
\begin{aligned}
& z_{2}^{+}=z_{2} \\
& \frac{\partial z_{2}^{+}(u)}{\partial u}=\delta+3\left[e^{\delta} x_{1}^{+}(u)+\left(1-e^{\delta}\right) u\right]^{2} \int_{0}^{\delta} e^{-2 \tau}\left(1-e^{-\tau}\right) d \tau \\
& +\left[e^{\delta} x_{1}^{+}(u)+\left(1-e^{\delta}\right) u\right]^{2} e^{-2 \delta}\left(1-e^{-\delta}\right) \\
& +6 u\left[\delta e^{\delta} x_{1}^{+}(u)+\left(1-e^{\delta}\right) u\right]\left[\int_{0}^{\delta} e^{-\tau}\left(1-e^{-\tau}\right)^{2} d \tau+\frac{e^{-\delta}\left(1-e^{-\delta}\right)}{3}\right] \\
& +3 u^{2}\left[\int_{0}^{\delta}\left(1-e^{-\tau}\right)^{3} d \tau+\frac{\left(1-e^{-\delta}\right)^{3}}{3}\right]
\end{aligned}
$$

with $z^{+}(u)=z_{2 k+1}$ and $x_{1}^{+}(u)=x_{1 k+1}$. The problem results in defining a digital I\&I control law which makes $z_{2} \rightarrow$ 0 as $k \rightarrow \infty$ preserving boundedness of the whole state trajectories (32)-(33). According to Section IV.2, one defines the control Lyapunov function $V\left(z_{2}\right)=z_{2}^{2}$ and the output $H\left(x_{1}, z_{2}, u\right)=L_{G_{z}\left(x_{1}, u\right)} V_{z}\left(z_{2}\right)$. It is a matter of computation to verify that zero-state detectability is inherited from the continuous-time one. At this point, one notices that in order to find $u=-\int_{0}^{1} H\left(x_{1}^{+}(s u), z_{2}^{+}(s u), s u\right) d s$ one has to solve a fifth degree equation in $u$, which is hard. Hence, one looks for the bounded I\&I negative output feedback defined as in Theorem 4.1. In particular, one computes

$$
G_{z}\left(F_{0}\left(x_{1}\right), 0\right)=\delta+3 x_{1}^{2} \int_{0}^{\delta} e^{-2 \tau}\left(1-e^{-\tau}\right) d \tau+x_{1}^{2} e^{-2 \delta}\left(1-e^{-\delta}\right)
$$

and sets $u=-\lambda\left(x_{1}, z_{2}\right) \varepsilon\left(x_{1}\right) z_{2} G_{z}\left(F_{0}\left(x_{1}\right), 0\right)$ choosing any $\mu>0$ and $\varepsilon\left(x_{1}\right) \lambda\left(x_{1}, z_{2}\right)$ so as to satisfy Theorem 4.1.

\section{A. Simulations}

Simulations are referred to the continuous-time I\&I feedback (red); its emulation (dotted red) which corresponds to hold constant over the sampling time the continuous-time controller; the dead beat approach (blue) and the bounded I\&I output feedback (dotted blue). The invariant set is plotted in black. The bounded feedback is implemented by choosing $\lambda(\cdot)$ as the upper-bound of (28). Simulations are reported for increasing values of the sampling period 

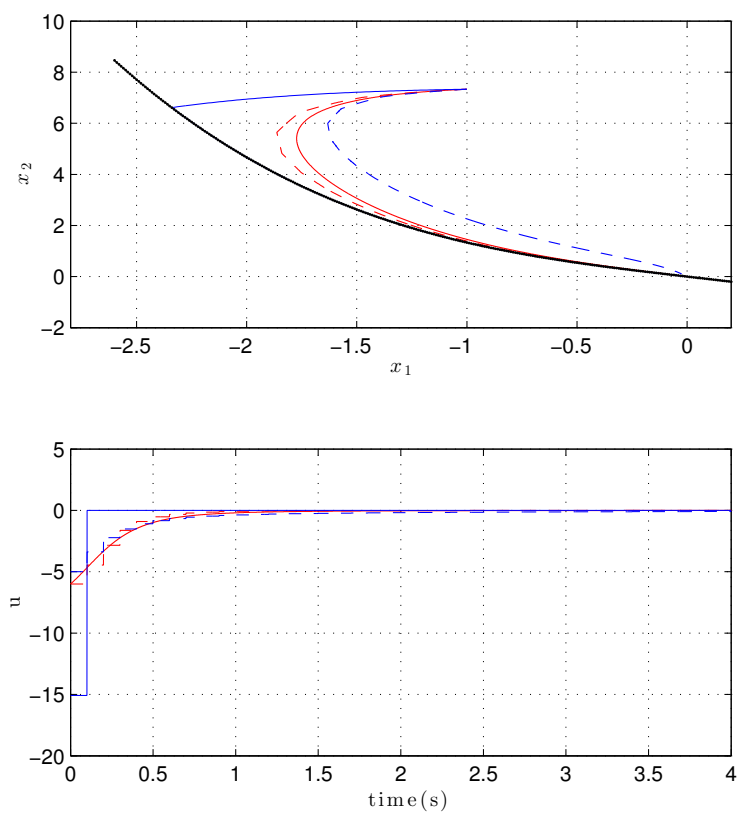

Fig. 1. $\delta=0.1$ seconds.
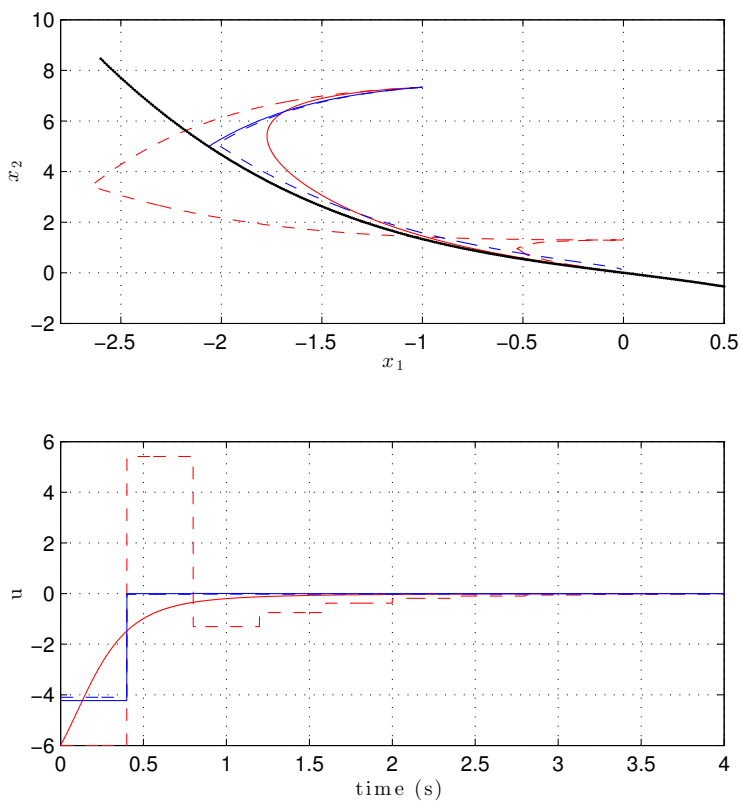

Fig. 2. $\delta=0.4$ seconds.

$\delta$ (0.1, 0.4 and 0.6 seconds). We can see that while the proposed controllers (dead-beat and average-passivity based) succeed in preserving the I\&I properties and the stability of the closed-loop equilibrium, the emulated-based control yields degradate performances for $\delta=0.4 s$ and instability for $\delta=0.6 s$. We also note that, as the sampling period increases, the sampled-data controllers require a lower control effort than the continuous-time and emulated-based ones.

\section{CONCLUSIONS}

With respect to usual forwarding, the proposed I\&I approach enables us to relax the knowledge of the $x_{1}$-control
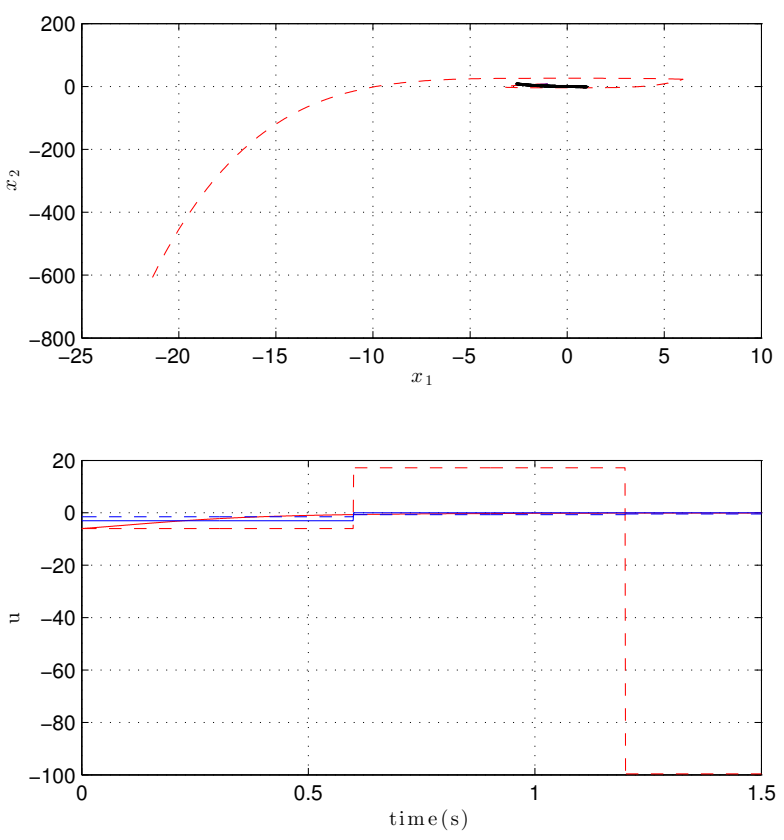

Fig. 3. $\delta=0.6$ seconds.

Lyapunov function $V$. Secondly, the bounded design relaxes the necessity to solve an implicit algebraic equality thanks to a suitable dynamic gain. Regarding sampled-data dynamics, it results that I\&I stabilization of continuous-time feedforward dynamics implies I\&I stabilization of its equivalent sampled-data dynamics in the case of two interconnected blocks.

\section{REFERENCES}

[1] M. Kristic, "Feedback linearizability and explicit integrator forwarding controllers for classes of feedforward systems," IEEE Transactions on Automatic Control, vol. 49, pp. 1668-1682, 2004.

[2] R. Sepulchre, M. Jankovic, and P. Kokotovic, "Integrator forwarding: a new recursive nonlinear robust control design," Automatica, vol. 33 , no. 979-984, 1997.

[3] P. Kokotović and M. Arcak, "Constructive nonlinear control: a historical perspective," Automatica, vol. 37, no. 5, pp. 637-662, 2001.

[4] F. Mazenc and H. Nijmeijer, "Forwarding in discrete-time nonlinear systems," vol. 71, no. 5, pp. 823-835, 1998.

[5] S. Monaco and D. Normand-Cyrot, "Forwarding stabilization of nonlinear discrete-time dynamics," in Proc. European Control Conference ECC'13, Zurich, 2013, pp. 2186-2192.

[6] A. Astolfi and R. Ortega, "Immersion and invariance: a new tool for stabilization and adaptive control of nonlinear systems," IEEE Transactions on Automatic Control, vol. 48, no. 4, pp. 590-606, 2003.

[7] A. Astolfi, D. Karagiannis, and R. Ortega, Nonlinear and adaptive control with applications. Springer Publishing Company, Incorporated, 2008

[8] Y. Yalcin and A. Astolfi, "Discrete time immersion and invariance adaptive control for systems in strict feedback form," in 50 th IEEECDC and EUCA-ECC, 2011, pp. 343-347.

[9] M. Mattioni, S. Monaco, and D. Normand Cyrot, "Digital stabilization of strict feedback dynamics through invariance and immersion," in Proc. IFAC MICNON, St Pétersbourg, 2015, pp. 1085-1090.

[10] "Nonlinear avarage passivity and stabilizing controller in discretetime," Systems \& Control Letters, vol. 60, pp. 431-439, 2011.

[11] F. Mazenc and L. Praly, "Adding integrations, saturated controls and stabilization for feedforward systems," IEEE Transactions on Automatic Control, vol. 41, no. 11, pp. 1559-1570, 1996.

[12] S. Monaco and D. Normand-Cyrot, "A unifying representation for nonlinear discrete-time and sampled dynamics," Journal of Mathematical Systems, Estimation and Control, vol. 5, no. 1, pp. 1-27, 1995. 